\title{
miR-152 as a tumor suppressor microRNA: Target recognition and regulation in cancer (Review)
}

\author{
XUEXIANG LIU*, JINWAN LI*, FENGXIAN QIN and SHENGMING DAI \\ Department of Laboratory Science, The Fourth Hospital Affiliated to Guangxi Medical University, \\ Liuzhou, Guangxi 545005, P.R. China
}

Received February 8, 2015; Accepted March 1, 2016

DOI: 10.3892/ol.2016.4509

\begin{abstract}
MicroRNAs (miRNAs or miRs) are endogenous translation repressors of protein-coding genes that act by binding to the 3'-untranslated region of their target genes, and may contribute to tumorigenesis by functioning as oncogenes or tumor suppressor genes. miR-152, a member of the miR-148/152 family, is aberrantly expressed in various diseases, including various types of cancer. A growing body of evidence has demonstrated that miR-152 may act as a tumor suppressor gene by regulating its target genes, which are associated with cell proliferation, migration and invasion in human cancer. In the present review, the gene structure and functions of miR-152 are discussed, and in particular, its regulatory mechanism, experimentally validated targets and tumor suppressor role in cancer, are highlighted.
\end{abstract}

\section{Contents}

1. Introduction

2. Biogenesis and evolution of miR-152

3. Experimentally validated targets of miR-152 in human cancer

4. miR-152 as a tumor suppressor miRNA in cancer

5. miR-152 in immune response

6. Therapeutic potential of miR-152 in cancer

7. Conclusion

Correspondence to: Professor Shengming Dai, Department of Laboratory Science, The Fourth Hospital Affiliated to Guangxi Medical University, 1 Liushi Road, Liuzhou, Guangxi 545005, P.R. China

E-mail: daishm@sina.com

${ }^{*}$ Contributed equally

Key words: miR-152, tumor suppressor, hypermethylation, human cancer

\section{Introduction}

MicroRNAs (miRNAs or miRs) are small, endogenous, non-coding RNAs that act as post-transcriptional regulators by binding to the 3'-untranslated region (UTR) of their target messenger (m)RNAs, resulting in the degradation of the mRNA or its translational inhibition (1-5).

miRNAs are 18-25 nucleotides in length, and are generated by cytoplasmic RNase III Dicer from 70-100 nucleotides-long endogenous hairpin pre-miRNA precursors (2-5). miRNAs have been highly conserved during evolution (6). Presently, $\sim 2,588$ human mature miRNAs have been deposited in the miRBase (release 21, http://www.miRBase.org/). Accumulating evidence indicates that miRNAs influence a variety of cellular functions, including proliferation, differentiation and apoptosis $(3,4,7)$. Furthermore, an increasing number of miRNAs have been implicated in a variety of diseases such as cancer (3). According to their function and expression pattern, miRNAs may act as oncogenes or tumor suppressors in cancer development and progression $(3,7)$.

miR-152 is one of the miRNAs that have attracted great interest in recent years, since it is implicated in various types of cancer $(8,9)$. Thus, it is of considerable significance to understand the regulation and function of miR-152 in human cancer. In the present study, the current knowledge of the functions of miR-152 in cancer is reviewed, with an emphasis on its regulation, targets and tumor suppressor role in human cancer.

\section{Biogenesis and evolution of miR-152}

miR-152 was first identified in mouse colon by tissue-specific cloning in 2002 (10). miR-152 is a member of the miR-148/152 family, which includes miR-148a, miR-148b and miR-152. Notably, in humans, the miR-152 gene is located on chromosome 17q21.32 (Fig. 1), within intron 1 of the coatomer protein complex, subunit zeta 2 (COPZ2) gene, and a $\mathrm{CpG}$ island is typically observed around its promoter region $(8,9,11)$. Following transcription and cleavage by Drosha, pre-miR-152 is transported to the cytoplasm, where it is further processed by Dicer to form a miR-152 duplex (5). Two different mature miR-152 sequences, namely miR-152-5p and miR-152-3p, appear to be excised from opposite arms of the miR-152 duplex (Fig. 2). miR-152-3p, which is excised from the $3^{\prime}$ arm 


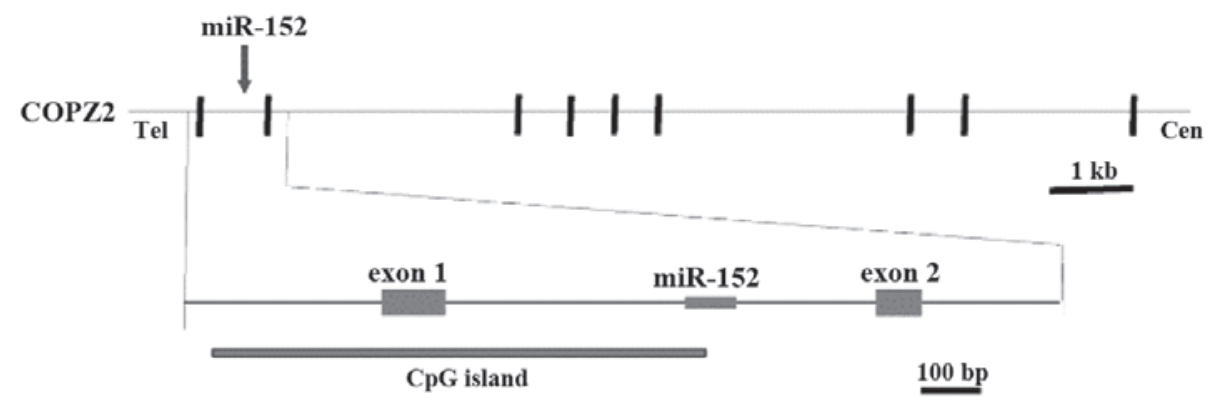

Figure 1. Structure of the genomic locus of human microRNA-152. The positions are designated according to the information in Epigenomics and Map Viewer for the coatomer protein complex, subunit zeta 2 gene, available at http://www.ncbi.nlm.nih.gov/gene/51226. miR, microRNA; COPZ2, coatomer protein complex, subunit zeta 2; Tel, telomere; Cen, centromere; kb, kilobase; bp, base pairs.

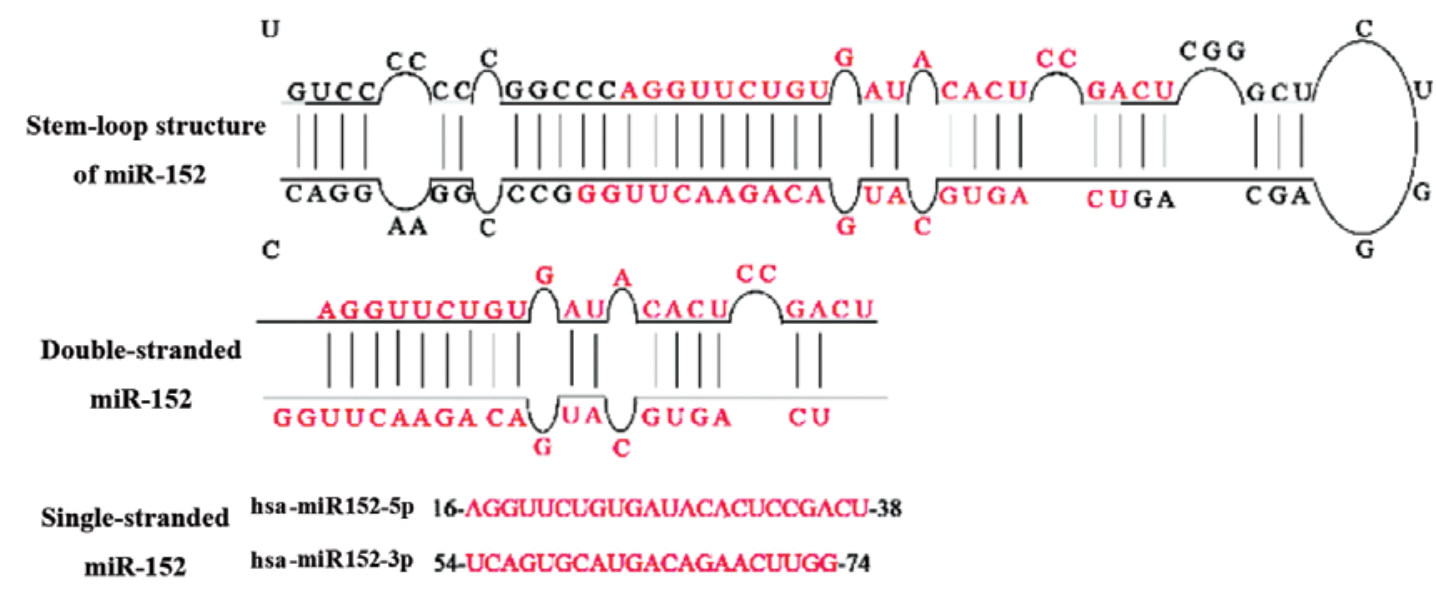

Figure 2. Biogenesis of miR-152. Two different mature miR-152 sequences, namely miR-152-5p and miR-152-3p, appear to be excised from opposite arms of the miR-152 duplex. miR, microRNA; hsa, Homo sapiens.

of the hairpin precursor, has been detected in more species than miR-152-5p.

The expression of miR-152 has been demonstrated in several species, according to miRBase (http://www.mirbase.org/). It is noteworthy that the mature miR-152 exhibits identical sequence in different species (with the exception of the extension at its $3^{\prime}$ end), and also shares an identical seed sequence across various species (Fig. 3A), which suggests that miR-152 is important in certain gene regulatory networks. To assess the degree to which miR-152 is conserved across species, a neighbor-joining (NJ) tree was constructed in 10 representative animal species by using genomic DNA sequences retrieved from miRBase (Fig. 3B). The miR-152 NJ tree clearly revealed the existence of two main lineages, one of which contains Homo sapiens, Canis familiaris, Ovis aries, Sus scrofa, Mus musculus, Bos taurus, Monodelphis domestica and Pan troglodytes, while the other lineage comprises Fugu rubripes and Danio rerio. These findings suggest that miR-152 is evolutionarily conserved and the recent lineage-specific miR-152 may be common to the old ancestral processor.

\section{Experimentally validated targets of miR-152 in human cancer}

The identification of miRNA targets and their regulatory sequences is a complex problem in miRNA research, and a considerable number of methods have been established to attempt such an identification, which are classified into computational (in silico) and experimental methods $(12,13)$. The prediction of miRNA targets using the current algorithms implemented in computational methods always results in a large number of false signals that do not reflect the situation in vivo; therefore, the predicted miRNA targets must be validated experimentally (12).

Hundreds of genes have been proposed as candidate targets of miR-152 with high scoring when predicted by computational programs such as PicTar (http://pictar.mdeberlin.de/) and TargetScan (http://www.targetscan.org/). A number of these genes have been further confirmed experimentally as targets of miR-152 (Table I). Braconi et al (14) first described that the DNA methyltransferase 1 (DNMT1) gene is a direct target of miR-148a and miR-152 by using luciferase reporter constructs, which revealed that miR-152 could target the 3'-UTR of DNMT1, resulting in a significant reduction of DNMT1 at both mRNA and protein levels. This finding was further confirmed in subsequent studies on ovarian cancer (15), endometrial cancer (9), nickel sulphide (NiS)-induced cell malignant transformation (16), breast cancer (17), hepatitis B virus-related hepatocellular carcinoma (18), pancreatic cancer (19) and prostate cancer (20). In addition, E2F transcription factor 3, mesenchymal to epithelial transition (MET), rapamycin-insensitive companion 


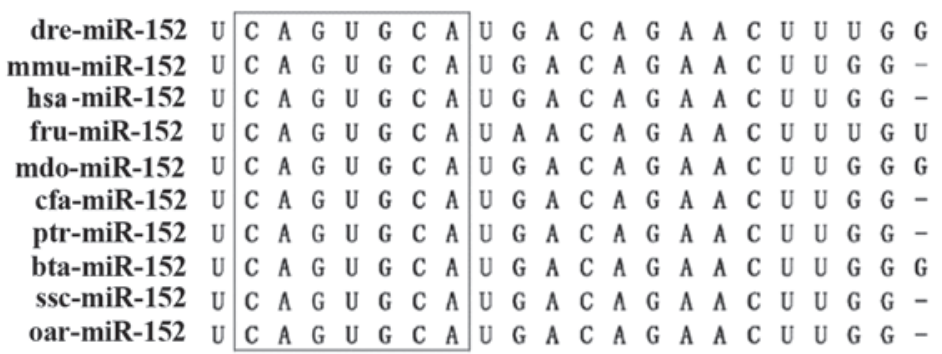

B

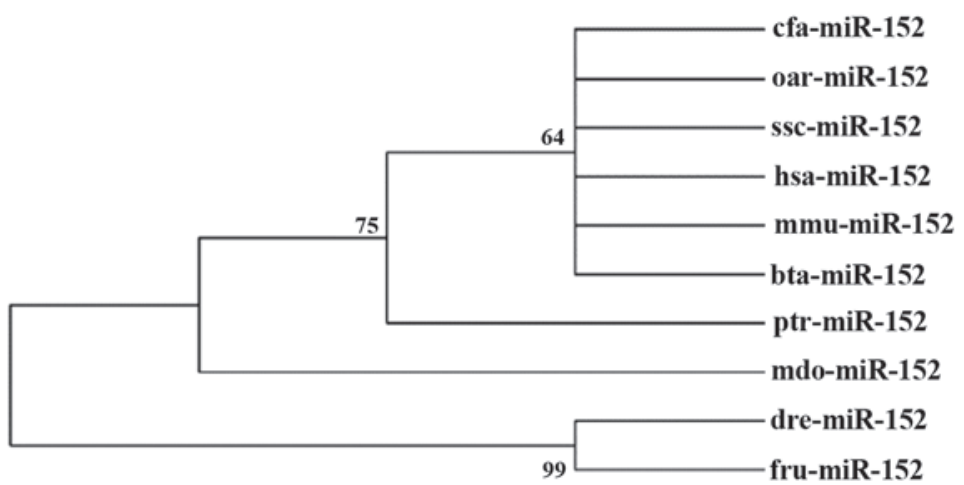

Figure 3. Conservation of miR-152 across species. (A) Sequence alignment of the mature miR-152 sequence in 10 different species. Seed sequences are highlighted in the black box. (B) Phylogenetic tree of miR-152. Numbers on each branch refer to the degree of reliability. miR, microRNA; hsa, Homo sapiens; cfa, Canis familiaris; oar, Ovis aries; ssc, Sus scrofa; mmu, Mus musculus; bta, Bos taurus; mdo, Monodelphis domestica; ptr, Pan troglodytes; fru, Fugu rubripes; dre, Danio rerio.

of mechanistic target of rapamycin (9), insulin-like growth factor 1 receptor (IGF-1R), insulin receptor substrate 1 (IRS1) (17), a disintegrin and metalloproteinase metallopeptidase domain 17 (ADAM17) $(21,22)$, Kruppel-like factor 4 (KLF4) (23), fibroblast growth factor 2 (FGF2) (24), winglessrelated integration site (Wnt1) (25), cluster of differentiation (CD) 151 (26), matrix metalloproteinase 3 (MMP3) (27) and transforming growth factor alpha (28) have been identified as targets of miR-152 in a wide array of human malignancies.

\section{4. miR-152 as a tumor suppressor miRNA in cancer}

Particular miRNAs may act as oncogenes or tumor suppressors depending on their expression pattern and function $(3,6)$. Those miRNAs with increased expression in tumor cells may be regarded as oncogenes, whereas downregulated miRNAs are considered as tumor suppressors (29).

There has been a rapid increase in the number of publications focusing on miR-152 in recent years, which revealed that the expression of miR-152 was inhibited in a variety of tumors, including ovarian (15), endometrial (9) and breast cancer (17). These findings suggest that miR-152 may potentially function as a tumor suppressor in human cancer. The main aim of the present review was to understand how miR-152 interacts with its target genes and to identify the potential role of miR-152 in cancer.

miR-152 mediates hypermethylation of DNA and its CpG island in human cancer. Aberrant DNA hypermethylation of tumor suppressor genes, global DNA hypermethylation (GDM) and disruption of histone modification patterns are the three most important epigenetic changes contributing to the malignant phenotype (30). In particular, DNA hypermethylation may be important in the initiation of multiple types of cancer $(30,31)$. Huang et al (18) first reported that inhibition of miR-152 could functionally result in GDM in hepatitis B virus-related hepatocellular carcinoma cell lines. Using liquid chromatography-mass spectrometry (MS)/MS, the authors identified that the overexpression of miR-152 reduced GDM from 6.31 to $4.08 \%$ in the HepG2 2.2.15 cell line, whereas miR-152 inhibitor increased GDM from 4.55 to $5.88 \%$ in HepG2 cells. Underexpression of miR-152 also increased the DNA methylation level of the promoter region of tumor suppressor genes such as glutathione S-transferase pi 1 and cadherin 1 in these cells (18). Azizi et al (19) demonstrated that the overexpression of miR-152 decreased GDM to normal patterns in pancreatic cancer cell lines and restored the expression of tumor suppressor genes, including B-cell lymphoma 2/adenovirus E1B $19 \mathrm{kDa}$ interacting protein 3 and secreted protein acidic and cysteine rich, by 3.8 and 2.9-fold, respectively. These data support a tumor suppressor role of miR-152 in the epigenetic aberration observed in cancer.

An increasing number of publications indicate that epigenetic silencing of tumor suppressor miRNAs by $\mathrm{CpG}$ island hypermethylation is a common feature of different types of human cancer $(31,32)$. Hypermethylation of the $\mathrm{CpG}$ island of miR-152 has been detected in $70(97.1 \%)$ cases of primary endometrial cancer (9). The concordance between DNA hypermethylation around the $\mathrm{CpG}$ island and underexpression of miR-152 was observed in $100 \%$ of the 70 cases of primary endometrial cancer (9). These results suggest that the hypermethylation of the $\mathrm{CpG}$ island of miR-152 may downregulate its expression, and may be involved in endometrial cancer. Due to the hypermethylation of its $\mathrm{CpG}$ island, silencing of 
Table I. Experimental confirmed targets of microRNA-152 in different types of cancer.

\begin{tabular}{|c|c|c|c|c|}
\hline Cancer & Expression & Biological process & Target gene & Refs. \\
\hline Endometrial cancer & Downregulation & Inhibited cell growth & $\begin{array}{l}\text { DNMT1, E2F3, MET, } \\
\text { RICTOR }\end{array}$ & (9) \\
\hline Cholangiocarcinoma & Downregulation & Reduced cell proliferation & DNMT1 & (14) \\
\hline Ovarian cancer & Downregulation & Reduced cell proliferation & DNMT1 & (15) \\
\hline $\begin{array}{l}\text { NiS-induced cell } \\
\text { malignant transformation }\end{array}$ & Downregulation & Inhibited cell growth & DNMT1 & (16) \\
\hline Breast cancer & Downregulation & $\begin{array}{l}\text { Inhibited cell proliferation, colony } \\
\text { formation and tumor angiogenesis }\end{array}$ & DNMT1, IGF-1R, IRS1 & (17) \\
\hline Hepatocellular carcinoma & Downregulation & Reduced cell proliferation & DNMT1, Wnt1 & $(18,25)$ \\
\hline Pancreatic cancer & Downregulation & Inhibited cell proliferation & DNMT1 & (19) \\
\hline Prostate cancer & Downregulation & Decreased cell growth, migration & DNMT1, TGF $\alpha$ & $(20,28)$ \\
\hline NSCLC & Downregulation & $\begin{array}{l}\text { Reduced cell proliferation, colony } \\
\text { formation, migration and invasion }\end{array}$ & ADAM17, FGF2 & $(22,24)$ \\
\hline Glioblastoma & Downregulation & $\begin{array}{l}\text { Reduced cell proliferation, migration, } \\
\text { invasion and pro-apoptosis }\end{array}$ & KLF4 & (23) \\
\hline Gastric cancer & Downregulation & Inhibited cell proliferation and motility & CD151 & (26) \\
\hline Glioma & Downregulation & $\begin{array}{l}\text { Reduced invasion } \\
\text { and invasion }\end{array}$ & MMP3 & (27) \\
\hline Neuroblastoma & Upregulation & $\begin{array}{l}\text { Increased neuroblast differentiation } \\
\text { and apoptosis }\end{array}$ & $\begin{array}{l}\text { CHUK, CUL5, } \\
\text { GADD45A }\end{array}$ & (36) \\
\hline
\end{tabular}

NSCLC, non-small cell lung cancer; NiS, nickel sulphide; DNMT1, DNA methyltransferase 1; E2F3, E2F transcription factor 3; MET, mesenchymal to epithelial transition; RICTOR, rapamycin-insensitive companion of mechanistic target of rapamycin ; KLF4, Kruppel-like factor 4; ADAM17, a disintegrin and metalloproteinase metallopeptidase domain 17; FGF2, fibroblast growth factor 2; IGF-1R, insulin-like growth factor 1 receptor; IRS1, insulin receptor substrate 1; Wnt1, wingless-related integration site 1; CD151, cluster of differentiation 151; MMP3, matrix metalloproteinase 3; TGF $\alpha$, transforming growth factor alpha; CHUK, conserved helix-loop-helix ubiquitous kinase; CUL5, cullin 5; GADD45A, growth arrest and DNA-damage-inducible, alpha.

miR-152 expression and overexpression of DNMT1 were also observed in NiS-transformed cells (16), breast cancer (17) and prostate cancer (20). Notably, there may be a crucial functional crosstalk between miR-152 and DNMT1 via a double-negative feedback regulatory loop, as speculated by Ji et al (16) regarding the classic 'chicken and egg' argument. DNMT1 exerts a crucial role in setting up and maintaining DNA methylation patterns in eukaryotic cells (33). Once increased expression of DNMT1 ('egg') occurs, DNMT1 is recruited to the miR-152 CpG island promoter, where it increases DNA methylation, contributing to reduced miR-152 expression ('chicken') (16). Furthermore, downregulated expression of miR-152 further increases DNMT1 expression by reduced targeting on DNMT1 3'-UTR (14-20). Therefore, epigenetic regulation of miR-152/DNMT1 may be important in tumorigenesis. In mixed lineage leukemia-rearranged infant acute lymphoblastic leukemia, hypermethylation of the $\mathrm{CpG}$ island of miR-152 was reported to be strongly correlated with a poor clinical outcome (34). Overall, hypermethylation of miR-152 may be considered as an epigenetic biomarker in human cancer.

miR-152 and its targets are associated with cell proliferation in cancer. miRNAs with antiproliferative and pro-apoptotic activity are likely to function as tumor suppressor genes (35). Antisense oligonucleotides targeting miRNAs have been used to identify miRNA functions (36). In those studies, the inhibition of miR-152 was observed to cause a decrease in cell growth in Hela cells. In neuroblastoma samples, the expression of miR-152 was upregulated, and miR-152 negatively controlled apoptosis by downregulating pro-apoptotic genes such as conserved helix-loop-helix ubiquitous kinase, cullin 5 and growth arrest and DNA-damage-inducible, alpha (37). By contrast, Zhou et al (9) reported that cell proliferation was remarkably inhibited by overexpression of miR-152 in ovarian cancer cells using 3-(4,5-dimethylthiazol-2-yl)-2,5-diphenyltetrazolium bromide assay. Therefore, whether miR-152 acts as a tumor suppressor gene remains controversial.

In previous studies, several miRNAs have been demonstrated to affect target genes that are involved in the control of cell proliferation and apoptosis (14-20). It has been well established that phosphatidylinositol-3 kinase (PI3K)/AKT and mitogen-activated protein kinase (MAPK)-mediated signaling pathways are two of the most predominant signaling pathways in human cancer, since they are involved in cell proliferation, survival and metabolism $(38,39)$. In breast cancer, overexpression of miR-152 significantly inhibited cell proliferation, colony formation and tumor angiogenesis by targeting IGF-1R and IRS1, and suppressing their downstream AKT and MAPK signaling pathways (17). KLF4 is a transcription factor that functions either as a tumor suppressor gene or as an oncogene in different contexts, and 
is critical for the control of essential cellular processes (40). In glioblastoma stem cells, miR-152 markedly inhibits cell proliferation, migration and invasion, and promotes cell apoptosis by targeting KLF4 (23). In addition, miR-152 could inhibit the expression of lectin, galactoside-binding, soluble, 3 by downregulating KLF4, thus attenuating the activation of the MAPK kinase $1 / 2$ and PI3K signaling pathway (23). In non-small cell lung cancer (NSCLC), Su et al (22) demonstrated that restoration of miR-152 significantly reduced cell proliferation, colony formation, migration and invasion partially via targeting ADAM17 (also known as tumor necrosis factor-alpha converting enzyme), which releases a variety of membrane-tethered proteins, the majority of which are associated with pathological processes such as cancer and inflammation (21). Another report revealed that the ectopic overexpression of miR-152 markedly inhibited NSCLC cell proliferation, promoted apoptosis, and suppressed migration and invasion through targeting FGF2 (22). CD151, a transmembrane protein of the tetraspanin family, participated in the mediation of tumor growth and metastasis (41). miR-152 was previously observed to be able to suppress the proliferation and motility of gastric cancer cell lines by targeting CD151 (26). In addition, miR-152 is also able to target Wnt1 and MMP3 (27) to inhibit cell proliferation in liver cancer cells, thus reducing glioma cell invasion and angiogenesis, respectively. Taking together, these findings suggest that miR-152 may modulate a variety of cellular processes such as cell proliferation, apoptosis and tumorigenesis via the regulation of its target genes and the tumor suppressor role of miR-152 in human cancer.

\section{5. miR-152 in immune response}

During the last 20 years, miRNAs have emerged as key regulators of a wide range of biological processes, including cell proliferation, differentiation, development and apoptosis $(3,4,7)$. Recent studies indicate that specific miRNAs are important in the immune system by modulating the development of immune cells and regulating the expression of genes that are critically involved in the immune response (42).

The innate immune system provides the first line of defence against infections and natural killer (NK) cells are critical mediators of the innate immune response (43). Human leucocyte antigen (HLA)-G is important in the cellular immune response, since it inhibits NK cell activity (44). miR-152 may downregulate the expression of HLA-G by directly targeting its 3'-UTR, leading to increased NK cell-mediated cytosis (45). Dendritic cells (DCs) are professional antigenpresenting cells, which bridge the innate and adaptive immune responses (45). Calcium/calmodulin-dependent protein kinase IIa (CaMKIIa), a major downstream effector of calcium signaling, regulates the critical stages of maturation and antigen-presentation capacity of human DCs (46). miR-152 is capable of inhibiting lipopolysaccharide-induced upregulation of major histocompatibility complex II expression and DC-initiated antigen-specific $\mathrm{CD}^{+} \mathrm{T}$ cell proliferation by targeting CaMKIIa (47). These findings suggest that miR-152 acts as a negative regulator in the immune system. Therefore, in addition to human cancer, miR-152 is also important in the innate immune response.

\section{Therapeutic potential of miR-152 in cancer}

Aberrant miRNA expression is a common feature of various types of human cancer, and miRNAs are crucial in the development of cancer $(3,7)$. As a result, numerous studies have focused on miRNA-based therapeutics, some of which are undergoing clinical trials in cancer patients (15). Targeting miRNAs may be used to control the growth of cancer cells, and also to enhance the efficacy of other therapies, such as reducing the drug resistance of tumors (48).

Resistance of cancer cells to chemotherapeutics is a clinical obstacle in the treatment of cancer patients (49). Cisplatin is the first-line chemotherapy drug for multiple malignancies (50). Xiang et al (15) demonstrated that miR-152 was involved in resistance to cisplatin in ovarian cancer. The authors confirmed that overexpression of miR-152 increased cisplatin sensitivity of SKOV3/DDP and A2780/DDP cells by inhibiting cell proliferation and promoting cell apoptosis via direct suppression of DNMT1. Therefore, miR-152 may serve as a therapeutic target for overcoming cisplatin resistance in ovarian cancer. This application is also likely to be used as a potential epigenetic therapeutic target in other types of cancer.

\section{Conclusion}

miR-152 is well conserved in evolution and possesses an identical seed sequence in different species. miR-152 may repress multiple target genes, a number of which have been validated by experimental methods. miR-152 binds to the 3'-UTR of its target genes, which are associated with different signaling pathways, thus leading to reduced cell proliferation and pro-apoptosis. In addition, miR-152 is involved in tumorigenesis, cell migration and invasion. miR-152 is located in the intron 1 of the COPZ2 gene, and is surrounded by a $\mathrm{CpG}$ island. Hypermethylation of the $\mathrm{CpG}$ island of miR-152 has been described in certain type of human cancer, and it may account for the downregulation of miR-152. These findings support the tumor suppressor role of miR-152 in human cancer, and suggest that miR-152 may serve as a prognostic biomarker and a therapeutic target in cancer patients.

However, the role of miR-152 in the progression of human tumors remains to be fully understood, particularly the mechanisms by which miR-152 contributes to tumorigenesis by binding to different target genes in different types of cancer. Further investigation on the function of miR-152 may lead to novel diagnostic and therapeutic approaches for the treatment of human cancer.

\section{Acknowledgements}

The present study was supported by Guangxi University of Science and Technology (Liuzhou, China; grant no. 2014G020403). The authors would like to thank Professor Wei Tian (Central South University, Changsha, Hunan, China) for his assistance in proofreading the present manuscript.

\section{References}

1. Filipowicz W, Bhattacharyya SN and Sonenberg N: Mechanisms of post-transcriptional regulation by microRNAs: Are the answers in sight? Nat Rev Genet 9: 102-114, 2008. 
2. Krol J, Loedige I and Filipowicz W: The widespread regulation of microRNA biogenesis, function and decay. Nat Rev Genet 11: 597-610, 2010

3. Chen CZ: MicroRNAs as oncogenes and tumor suppressors. N Engl J Med 353: 1768-1771, 2005.

4. Bartel DP: MicroRNAs: Genomics, biogenesis, mechanism, and function. Cell 116: 281-297, 2004.

5. Pasquinelli AE: MicroRNAs and their targets: Recognition, regulation and an emerging reciprocal relationship. Nat Rev Genet 13: 271-282, 2012.

6. Garzon R, Calin GA and Croce CM: MicroRNAs in cancer. Annu Rev Med 60: 167-179, 2009.

7. Jansson MD and Lund AH: MicroRNA and cancer. Mol Oncol 6: 590-610, 2012

8. Lagos-Quintana M, Rauhut R, Yalcin A, Meyer J, Lendeckel W and Tuschl T: Identification of tissue-specific microRNAs from mouse. Curr Biol 12: 735-739, 2002.

9. Zhou X, Zhao F, Wang ZN, Song YX, Chang H, Chiang Y and Xu HM: Altered expression of miR-152 and miR-148a in ovarian cancer is related to cell proliferation. Oncol Rep 27: 447-454, 2012.

10. Chen Y, Song YX and Wang ZN: The microRNA-148/152 family: Multi-faceted players. Mol Cancer 12: 43, 2013.

11. Tsuruta T, Kozaki K, Uesugi A, Furuta M, Hirasawa A, Imoto I, Susumu N, Aoki D and Inazawa J: miR-152 is a tumor suppressor microRNA that is silenced by DNA hypermethylation in endometrial cancer. Cancer Res 71: 6450-6462, 2011.

12. Ekimler S and Sahin K: Computational methods for microRNA target prediction. Genes (Basel) 5: 671-683, 2014.

13. Thomson DW, Bracken CP and Goodall GJ: Experimental strategies for microRNA target identification. Nucleic Acids Res 39: 6845-6853, 2011.

14. Braconi C, Huang N and Patel T: MicroRNA-dependent regulation of DNA methyltransferase-1 and tumor suppressor gene expression by interleukin- 6 in human malignant cholangiocytes Hepatology 51: 881-890, 2010.

15. Xiang Y, Ma N, Wang D, Zhang Y, Zhou J, Wu G, Zhao R, Huang $\mathrm{H}$, Wang $\mathrm{X}$, Qiao $\mathrm{Y}$, et al: miR-152 and miR-185 co-contribute to ovarian cancer cells cisplatin sensitivity by targeting DNMT1 directly: A novel epigenetic therapy independent of decitabine. Oncogene 33: 378-386, 2014

16. Ji W, Yang L, Yuan J, Yang L, Zhang M, Qi D, Duan X, Xuan A, Zhang W, Lu J, et al: MicroRNA-152 targets DNA methyltransferase 1 in NiS-transformed cells via a feedback mechanism. Carcinogenesis 34: 446-453, 2013.

17. Xu Q, Jiang Y, Yin Y, Li Q, He J, Jing Y, Qi YT, Xu Q, Li W, $\mathrm{Lu}$ B, et al: A regulatory circuit of miR-148a/152 and DNMT1 in modulating cell transformation and tumor angiogenesis through IGF-IR and IRS1. J Mol Cell Biol 5: 3-13, 2013.

18. Huang J, Wang Y, Guo Y and Sun S: Down-regulated microRNA-152 induces aberrant DNA methylation in hepatitis B virus-related hepatocellular carcinoma by targeting DNA methyltransferase 1. Hepatology 52: 60-70, 2010.

19. Azizi M, Teimoori-Toolabi L, Arzanani MK, Azadmanesh K, Fard-Esfahani P and Zeinali S: MicroRNA-148b and microRNA-152 reactivate tumor suppressor genes through suppression of DNA methyltransferase-1 gene in pancreatic cancer cell lines. Cancer Biol Ther 15: 419-427, 2014.

20. Theodore SC, Davis M, Zhao F, Wang H, Chen D, Rhim J, Dean-Colomb W, Turner T, Ji W, Zeng G, et al: MicroRNA profiling of novel African American and Caucasian prostate cancer cell lines reveals a reciprocal regulatory relationship of miR-152 and DNA methyltranferase 1. Oncotarget 5: 3512-3525, 2014

21. Wu Y, Huang A, Li T, Su X, Ding H, Li H, Qin X, Hou L, Zhao Q, Ge X, et al: miR-152 reduces human umbilical vein endothelial cell proliferation and migration by targeting ADAM17. FEBS Lett 588: 2063-2069, 2014.

22. Su Y, Wang Y, Zhou H, Lei L and Xu L: MicroRNA-152 targets ADAM17 to suppress NSCLC progression. FEBS Lett 588: 1983-1988, 2014

23. Ma J, Yao Y, Wang P, Liu Y, Zhao L, Li Z, Li Z and Xue Y: miR-152 functions as a tumor suppressor in glioblastoma stem cells by targeting Krüppel-like factor 4. Cancer Lett 355: 85-95, 2014.

24. Cheng Z, Ma R, Tan W and Zhang L: miR-152 suppresses the proliferation and invasion of NSCLC cells by inhibiting FGF2 Exp Mol Med 46: e112, 2014

25. Huang S, Xie Y, Yang P, Chen $P$ and Zhang L: HCV core protein-induced down-regulation of microRNA-152 promoted aberrant proliferation by regulating Wnt1 in HepG2 cells. PLoS One 9: e81730, 2014.
26. Zhai R, Kan X, Wang B, Du H, Long Y, Wu H, Tao K, Wang G, Bao L, Li F and Zhang W: miR-152 suppresses gastric cancer cell proliferation and motility by targeting CD151. Tumour Biol 35: $11367-11373,2014$

27. Zheng X, Chopp M, Lu Y, Buller B and Jiang F: miR-15b and miR-152 reduce glioma cell invasion and angiogenesis via NRP-2 and MMP-3. Cancer Lett 329: 146-154, 2013.

28. Zhu C, Li J, Ding Q, Cheng G, Zhou H, Tao L, Cai H, Li P, Cao Q, $\mathrm{Ju}$ X, et al: $\mathrm{miR}-152$ controls migration and invasive potential by targeting TGF $\alpha$ in prostate cancer cell lines. Prostate 73 : 1082-1089, 2013

29. Dalmay T and Edwards DR: MicroRNAs and the hallmarks of cancer. Oncogene 25: 6170-6175, 2006.

30. Lujambio A and Esteller M: $\mathrm{CpG}$ island hypermethylation of tumor suppressor microRNAs in human cancer. Cell Cycle 6: 1455-1459, 2007.

31. Plass C: Cancer epigenomics. Hum Mol Genet 11: 2479-2488, 2002.

32. Esteller $\mathrm{M}: \mathrm{CpG}$ island hypermethylation and tumor suppressor genes: A booming present, a brighter future. Oncogene 21: 5427-5440, 2002.

33. Veeck J and Esteller M: Breast cancer epigenetics: From DNA methylation to microRNAs. J Mammary Gland Biol Neoplasia 15: 5-17, 2010.

34. Stumpel DJ, Schotte D, Lange-Turenhout EA, Schneider P, Seslija L, de Menezes RX, Marquez VE, Pieters R, den Boer ML and Stam RW: Hypermethylation of specific microRNA genes in MLL-rearranged infant acute lymphoblastic leukemia: Major matters at a micro scale. Leukemia 25: 429-439, 2011

35. Hwang HW and Mendell JT: MicroRNAs in cell proliferation, cell death, and tumorigenesis. Br J Cancer 94: 776-780, 2006.

36. Cheng AM, Byrom MW, Shelton J and Ford LP: Antisense inhibition of human miRNAs and indications for an involvement of miRNA in cell growth and apoptosis. Nucleic Acids Res 33: 1290-1297, 2005

37. Ragusa M, Majorana A, Banelli B, Barbagallo D, Statello L, Casciano I, Guglielmino MR, Duro LR, Scalia M, Magro G, et al: MIR152, MIR200B, and MIR338, human positional and functional neuroblastoma candidates, are involved in neuroblast differentiation and apoptosis. J Mol Med (Berl) 88: 1041-1053, 2010.

38. Vivanco I and Sawyers CL: The phosphatidylinositol 3-kinase AKT pathway in human cancer. Nat Rev Cancer 2: 489-501, 2002.

39. Chang L and Karin M: Mammalian MAP kinase signalling cascades. Nature 410: 37-40, 2001.

40. Rowland BD and Peeper DS: KLF4, p21 and context-dependent opposing forces in cancer. Nat Rev Cancer 6: 11-23, 2006.

41. Suzuki S, Miyazaki T, Tanaka N, Sakai M, Sano A, Inose T, Sohda M, Nakajima M, Kato H and Kuwano H: Prognostic significance of CD151 expression in esophageal squamous cell carcinoma with aggressive cell proliferation and invasiveness. Ann Surg Oncol 18: 888-893, 2011.

42. Hoefig KP and Heissmeyer V: MicroRNAs grow up in the immune system. Curr Opin Immunol 20: 281-287, 2008

43. Beaulieu AM, Bezman NA, Lee JE, Matloubian M, Sun JC and Lanier LL: MicroRNA function in NK-cell biology. Immunol Rev 253: 40-52, 2013.

44. Manaster I, Goldman-Wohl D, Greenfield C, Nachmani D, Tsukerman P, Hamani Y, Yagel S and Mandelboim O: MiRNAmediated control of HLA-G expression and function. PLoS One 7: e33395, 2012

45. Zhu XM, Han T, Wang XH, Li YH, Yang HG, Luo YN, Yin GW and Yao YQ: Overexpression of miR-152 leads to reduced expression of human leukocyte antigen-G and increased natural killer cell mediated cytolysis in JEG-3 cells. Am J Obstet Gynecol 202: 592.e1-592.e7, 2010.

46. Herrmann TL, Agrawal RS, Connolly SF, McCaffrey RL, Schlomann J and Kusner DJ: MHC Class II levels and intracellular localization in human dendritic cells are regulated by calmodulin kinase II. J Leukoc Biol 82: 686-699, 2007.

47. Liu X, Zhan Z, Xu L, Ma F, Li D, Guo Z, Li N and Cao X: MicroRNA-148/152 impair innate response and antigen presentation of TLR-triggered dendritic cells by targeting CaMKII $\alpha$. J Immunol 185: 7244-7251, 2010.

48. Nana-Sinkam SP and Croce CM: MicroRNA dysregulation in cancer: opportunities for the development of microRNA-based drugs. IDrugs 13: 843-846, 2010.

49. Mellor HR and Callaghan R: Resistance to chemotherapy in cancer: a complex and integrated cellular response. Pharmacology 81: 275-300, 2008.

50. Harries M and Gore M: Part I: Chemotherapy for epithelial ovarian cancer-treatment at first diagnosis. Lancet Oncol 3: 529-536, 2002. 